\title{
Cultural Addiction and Ritualistic Agreements Regarding Family Engagements Working Roles and Social Agreements Made Thereof
}

\author{
Tumanako William Hongi Pugh* \\ Mental Health Community Leader and Role Model for the Peer Support Community, Australia
}

*Corresponding author: Tumanako William Hongi Pugh, Mental Health Community Leader and Role Model for the Peer Support Community, Australia.
Received Date: January 04, 2019

Published Date: January 23, 2019

\begin{abstract}
Cultural addiction and ritualistic agreements regarding family engagements, working roles and social agreements made thereof details the nature of a culture of addiction equating across human and animal communities and also environments yet how such cultural and social environments impact upon individual decision making processes regarding addiction, tastes for pleasure and such social agreements agreed there upon by a cultural value put to the herd mentality, leadership roles, team building and the family dynamic governing groups and each of our own individual taste and appetite related to addiction and such habitual agreements made thereof yet meanwhile the engagement furthermore of the recovery of communities, cultures and environments by a collective of individuals yet in articulation in regards to how such a relationship exists within human society and nature.
\end{abstract}

Of which relates to rehabilitation centres, early intervention centres, prisons, gaols, mental health facilities and recovery based communities alike unto Peer Support and how such models equate inclusively across the animal kingdom regarding rehabilitation, addiction, illness, impairment, toxic environments and those humanitarian social agreements made by contrast endorsing, implementing, initiating and empowering a recovery based community governing individuals by a fraternity and a recovery based institution gathered around reducing recidivism, relapse and institutionalisation yet meanwhile empowering those impacted individuals with hope to re-integrate back into society and/or back into nature or the wild.

By a health model driving mental health as being one of abstinence and recovery activation into recovery and the community engagement processes thereof empowering themselves and others through connectivity, hope, empowerment, personal choice, commonality and story-telling shared rites of passage yet meanwhile the initiation processes into L.O.R.E and the LAW entirely as one.

Regarding the recovery activation processes and community engagements that one such individual may consider time after time by engaging in the community engagement processes in personal empowerment in further self-ownership, self-validation, selfempowerment, self-analysis, self-understanding, self-awareness, self-knowingness and self-reflection yet meanwhile utilising the creativity of the mind regarding the Arts and Education to rise out of the shadows and into the light of a new days dawn as recovered lives with hope therefore as always endorsing Psychological Models of mind, body, soul and spirit whilst meanwhile incorporating the dynamic nature of the individual.

\section{The Nature of a Culture of Addiction Equating} Across Nature and Human Animal Based Cultures, Environments Meanwhile Society as a Whole

There is an intrinsic cultural nature of intoxication and addiction imbedded into nature and society including each of our own individually tailored planned and programmed realities that the inherent tendency for the need for animals and living populations to suite one another by suiting ourselves by reflection to our peer representative populations complementing each other regarding its' own species, genus and phylum density populations of living forms, living conditions and environments relative to all living entities to modify each of their own selves, cultures, populations and each of our own collective environments due to the battle of the self or individual species regarding a competitiveness for food and survival mechanisms imbedded into mother nature and the battle between density populations, environments, life streams, cultures and living organisms. 
Due to populations and cultures of disparage, such as species, genus and phylum and finally its' relationships with each of our own ritualistic behavioural relationships that we engage with regarding other population disparage including each of our own cultural environments and the ritualistic agreements we make between a system and/or network, such as in Human Populations being as Family, Working roles and those Social engagements we engage in partnership with of which can be reflected in nature by the consumption of a food source naturally designed to be consumed by a particular species even if such a food source is an intoxicant such as is reflected in the animal kingdom in regards to birds, apes, monkeys and insects [1].

Regarding rules, regulations, modus operadus and behavioural types relating to how we view, understand and engage with Family such as phylum, Working Families such as genus and Friends such as species and all lower class species populations regarding social interactions within the network and how things play out yet meanwhile relaying through teamwork and the competitiveness of a harsh environment to build an established behavioural, environmental and cultural structure of toxicity populations within the stream and the addictive tendency for such addictive cultural toxic environments to thrive upon the available source of a culture of addictive toxic all-consuming substances and the damaging effects of over-consumption in consuming large volumes of relatively dense matter regarding a food source of nourishment and/or pleasure sports food and intoxicant sources.

Thereby decreasing longevity of such planetary living life forms such as macrocosmic and microcosmic laws at work here in due time only resulting in the purging of that which is the blight such as the individual and species, working environments and genus whilst also interacting with the greater family and phylum to therefore meanwhile re-calibrate and ever-evolve due to the nature of Darwinism and the ever evolution of life forms throughout the universe as universal law.

In that the nature of a culture of addiction is inherently built into universal biological laws to survive against all competitive odds in nature, consume a food source of nourishment such as chemicals, sunlight and substances bringing all life sustaining energy yet meanwhile propagating itself to propagate the species in ever growing populations bringing a density of living organisms and/ or culture of living entities or otherwise chain reactions governed by competition, survival, growth and density of propagation populations, consumption and evolution to therefore satisfy the nature of all living life forms and the propagation of themselves to therefore satisfy their own universal pre-programmed disposition of self-sustainability and propagation brought about by the nature of universal laws such as evolution and Darwinism [2].

Therein contained through biological and chemical means governing population densities and toxicity of environmental cultures relative to the consumable food source or otherwise poisonous environments and ecosystems of which eventually brings degradation of cultures and environments, poisoning of lifeforms, a critical mass to be reached therefore without the ceasing of an experiment to be simply put that all living cultures are but only experiments, meanwhile thereby, purging all toxic life-forms through cleansing throughout planetary or otherwise a purging of physiological ecology.

Therefore by a re-calibration and a further evolution of the species, genus and phylum to evolve and then bring about balance and harmony within the environment governed by cleansing of which therefore sustains life and enables further growth to seed of which it seems that due to the nature of toxic environments in purification of all-consuming consumption of resources that a nature of cultural addiction seems to be imbedded into mother nature and societies as one by a pre-programmed disposition of a cultural cleansing of putrid environments.

In that by an agreement of consumption related to a ritualistic and cultural agreement made by networks, families and cultures of species impacts upon each of our own independent and isolated environments relative to culture and cultural conditioning to then therefore cease to maintain each of our own individual individualism and identity relating to the individual expressing itself in a system that seeks by inherent rules conformity, normalcy and balance by one of competition against an isolated foothold finding growth against the odds stacked against itself and all living entities from birth.

Due to the influence of peer cultural influences to then remain connected to such peer and environmental controls regarding addiction and addictive environments governed by one of maintaining the addiction gathered around peer influence yet individualistic by nature and unique all throughout of which only finally manages the planetary earth to ever-evolve whilst meanwhile weighs upon how such impacts upon each of our own individual eco-decisions related to individualism and expressionism regarding social and family agreements of conformity due to a culture of environmental factors relating to influences made by the peer group family dynamic, peer working forces and the social agreements made therein upon individual eco-decision making processes as being mind, body, soul and spiritual decisions regarding wellbeing and wealth or otherwise ill-health and addiction regarding an illness of an addictive personality regarding his or her ill and impaired viewpoints of addiction.

That furthermore by understanding the growth of a species declining into death and extinction yet by the exiting of a potentially endangered species from a toxic environment in further damaging a dying population into a rehabilitation environment will determinately provide a suitable conducive environment for better growth, re-learning, re-programming, fine-tuning, safety, recalibration and possibly more so often than not re-integration into the community and environment.

To therefore grow, alter each of their own destiny to then thereby unlearn to re-learn, continue to live and survive with resilience in a more wholesome environment without toxic alllike tick consuming bug-like addictive substances, poisons and environments such as poachers or influential peers of toxic related poly-substance abuse utilising their potent toxic influence over such peers whom desire to sustain abstinence. 
Yet meanwhile at the same time such treatments of a possible extinction of a species can also be seen in human addiction and recovery programs such as the recovery of an individual through the use of conducive environments such as rehabilitation and early intervention centres to provide a conducive environment for recovery given certain constraints and reward systems apparent with hope to recover fully of which is more than applicable to provide the sustained recovery of individuals such as exiting the current environmental controls sustaining such addictions such as toxic home environments, peer pressure from friends and work colleagues, toxic partnerships and toxic relationships in codependence.

Whilst placing controls over the management of one's own recovery activation regarding accessibility of toxic friends or toxic family members to neither influence the program nor sustain control over participants yet place safety controls over participants regarding relapse by one of re-education, rehabilitation, the twelve steps, cutting all ties with toxic influences and reintegration gathered around personal choice, personal ownership, empowerment and hope in recovery activation.

To therefore re-integrate into society gathered around making life changing life style changes to alter the course of one's destiny.

In that such a recovery application of model is also possible to be achieved in mental health facilities, gaols and prisons yet maybe a considerable determining factor off setting relapse yet if fine-tuned and tweaked such clinical facilities and clinical recovery models if applied through the right application regarding improved social settings and social environments relative to providing natural settings, mural story-telling spaces and special spaces to bring hope in those places of hopelessness such as in those prisons, gaols and mental health facilities.

To therefore build human resilience, hope, empowerment, growth and learning governed around recovery, abstinence, maintaining duties and working roles, family obligations and the social governance that a holistic recovery of individuals, communities and peoples may be more likely to be achieved therefore expressing ourselves in each of our own collective lives as unique individuals expressing ourselves dynamically yet empowered by hope and education, that such issues as recidivism and institutionalisation may be reduced by the re-education of such individuals and re-integration methods governed by a personal choice in recovery activation, rehabilitation and hope by guiding such individuals through support and tailored mentoring programs may such sustainable recovered lives with hope be achieved of which is essential.

That simply by being placed into a mental health facility and/ or gaol will never ensure one's personal rehabilitation and reintegration into the community in that only by personal ownership of being powerless whilst having hope by personal choice to recover to therefore enter into a recovery activation process by personal choice in that such an abstinence and re-integration may be so of which is essential to undertake the recovery processes and yet without hope there is none yet meanwhile without personal ownership and personal choice to recover there is little to no hope whatsoever at all of recovery.

In that potentially any substance, engagement, medium, working role and obsession may be considered to be an addiction regarding an addictive personality engaging in something that potentially may harm oneself due to such habitual pleasure seeking tastes for a substance and/or activity of pleasure yet bring about a burn out, nervous break-down and further spiral into deterioration and eventual death and/or suicide.

In that the nature of addiction is holistically altogether defined as anything that may be uncontrollably out of control yet meanwhile is consistently damaging to each of our own individual and/or collective well-being regarding each of our own individual collective psyche to neither be able to engage in coherent thought processing and/or physical and psychological control over each of our own individual taste for such substances, food sources, mediums, working engagements, sexual appetites and those uncontrollable impulsive behaviours relating to addiction, habitual pleasure and satisfying such pleasures [3] in that all addictions should be negated by abstinence or otherwise controlled entirely by a model of moderation.

Ritualistic Agreements Regarding Familiar Social Engagements and Family Obligations Relating To Duty, Service, Working Roles and Socialising Engagements In Regards to A Culture Of Addiction and The Nature Thereof

Ritualistic Agreements are the agreements we make when in a social or familiar setting regarding each of our own individual interactions with family, work colleagues and socialising parties and how such may impact upon our own individual well-being regarding addiction and/or otherwise those particular activities that we each undertake in each of our own collective lifestyles of which therefore impacts upon each of our own individual lifestyle choices relating to such a taste for either substance use and/ or abuse, alcohol consumption, tobacco smoking yet meanwhile includes those such things as food, beverages, work, fame and even sex.

In that such a culture of socialising agreements made by either family, work colleagues and/or friends are present in all societal decision making processes and therefore contributes to the promotion of addiction and the relationships we have with friends and family yet meanwhile impacts upon each of our own individual decision making processes to make coherent, powerful and positive decisions for each of our own selves regarding peer pressure and such socialising agreements made due to those particulars tastes we have individually decided upon using, such as drugs, sex, money, food and even the addiction for the taste for fame, glory seeking, adulation, admiration and worship.

And therefore such an agreement made by ourselves only promotes each of our own individual death and demise gathered around social agreements based upon ritualistic activities, peer group pressure and the self-sustaining of an image based society, addiction and the promotion of illness. 
Of which impairs individuals to make decisions for themselves yet meanwhile only allows the herd mentality [4] to sustain its' own by a herd value put to the leadership of such a herd mind gathered around suiting the herd's sole purpose to procreate itself yet meanwhile discards individualism and the individual to express themselves openly and honestly within such a conformist herd mentality due to the nature of leadership and a sheep-like mentality of follow the leader even to one's own demise and possible doom that may ensue by such a herd minded mentality of follow the leader.

Therefore such a herd mentality only seeks to dominate herds and others towards conforming to the herd ideal of such social party agreements and arrangements made by a leadership value based around ritualistic activities and ritualistic agreements of being herded and lead into such agreements and socialising parties gathered by social image, a taste for addiction (whatever that is) and such agreements made by family, leaders, friends, peers and colleagues of which therefore only isolates the group dynamic further into sub-groups and social groups of social domination.

In that by an ideal based around socialising party agreements determines the rules and conditions of the herd ideal and such a party ideal regarding what tastes a party agreement agrees to and the relationship individuals have with each of their own individual appetite and taste yet meanwhile agreeing to the group and herd dynamic regarding lifestyle due to familiar and family environments detailing such things through child rearing programs and socialising agreements and arrangements made beforehand yet based around such family based dynamics and family agreements governed around strengthening relationships may alter individuals lives and views due to herds, herd dynamics and a herd mentality of following blindly the 'leader one' mentality.

Of which due to the family dynamic regarding each of our own collective or individual child rearing programs and those lessons learnt as children, teens and adults pre-determine such party agreements which rely solely upon family dynamics in leadership roles to be lead and sustain themselves as a family and group dynamic gathered around team work, team building and leadership regarding strengthening bonds, friendship and the greater family building of networking and family.

As family dynamics and family operations are the primary determinant of all outcomes due to the relationship between masculine/feminine principles regarding yin and yang principles and leadership of which is meanwhile governed by human relationships, family principles and family values yet will furthermore pre-determine each of our own individual and (yet possibly?) collective outcomes governed around child rearing programs, learned behaviours and pre-disposed dispositions learnt from ancestral generational family patterns that have persisted throughout time eternal.

In that by a value put to teamwork, leadership positions and the nature of building a team environment yet meanwhile relating to how we each individually view each of our own individual tastes for certain delights during such family orientated team building engagements and/or obligations put to the family dynamics and team building endeavours to then strengthen bonds between family members and/or otherwise socialising agreements made at work between colleagues or furthermore those social agreement made when engaging with friends will thereby be determined by each of our own individual viewpoints and family relationships with parents and siblings in child rearing programs.

Of which also strengthens bonds and friendships between one another yet meanwhile dynamically influences how the group dynamic views such tasteful delights as being treats for offer whilst engaging in also each of our own views and self-sustained participation in such appetites, either behind closed doors or otherwise openly, in regards to how such treats and tastes for either substances and/or those addictive delights for pleasuring ourselves may or may not be consumed.

Gathered around our individual views about such things and the group dynamic ideals put to each of our own appetites for such delights of taste whether refined or not in that the pre-disposition is put to leadership, team-building, team work and a family dynamic of which primarily is gathered around building an immediate family, greater family and friendship between partners, colleagues, siblings and friends or otherwise groups and/or communities.

That by a principle put to sharing from commonality in sharing common experiences felt between others in group dynamic operations that such bonds may be strengthened, enhanced, refined and further worked upon as a work in progress regardless of how the environment may be toxic or otherwise healthy due to the nature of caring for one's own and the group building dynamics governed around team work and the valuing of one another as important.

In finally stating that by contrast to rehabilitation services, gaols and mental health facilities that Peer Support is a community based recovery activation program governed by role modelling and friendship yet altogether as one is not just friendship alone but it is a valued relationship gathered around breaking down barriers of isolation and disenfranchisement due to mental health issues, mental illness, drug addiction, homelessness, poverty and further disempowerment by a common thread of commonality in sharing stories and role-modelling what it is like to be healthy, sustain one's well-being, maintain life for the long term, remain obstinate, build human resilience and rise out of the shadows of disenfranchisement.

By a value put role-modelling by example yet meanwhile supporting those peers holistically through the empowerment of hope, sharing stories of commonality, education, self-ownership and a connection to the I self in self-knowingness, self-awareness, self-reflection, self-validation and self-analysis to thereby break down barriers in those individual peers lives we role model with as a tailored plan therefore empowering such peers altogether by connectivity, community and hope that human resilience may be sustained and a gradual steady rise may occur into health, mental health, well-being, wellness and socio-economic prosperity [5].

In that Peer Support is viewed as being a relatively new field of mental health yet the support that may be provided for the community as a whole governed by recovery activation, role- 
modelling, human resilience, support, empowerment, education, the arts, the community and community engagement processes, inclusivity and hope is essential to recover peoples, individuals and communities altogether as one.

\section{Acknowledgement}

None.

\section{Conflict of Interest}

Author declare no conflict of interest.

\section{References}

1. Muller N (2011) Animals getting high: 10 common drunks. Australian Geographic.

2. (2018) Darwins Theory of Evolution.

3. Horvath T, Misra K, Epner AK, Copper GM (2018) Definition of Addiction.

4. Nauert R (2016) Herd Mentality Explained. Psych Central.

5. Pugh TWH (2018) The Australian Peer Support Mandate ( $2^{\text {nd }}$ edn). Austin McCauley, London, pp. 1-132. 